\title{
Caracterización del Ñeque, Bebida Alcohólica elaborada Artesanalmente en la Costa Caribe Colombiana
}

\author{
Diego F. Tirado*, Diofanor Acevedo, Piedad M. Montero \\ Universidad de Cartagena, Facultad de Ingeniería, Programa de Ingeniería de Alimentos, Grupo de \\ Investigación NUSCA, Avenida el Consulado, Calle 30 No. 48-152. Cartagena, Bolívar-Colombia \\ (e-mail: dtiradoa@unicartagena.edu.co) \\ * autor a quien debe ser dirigida la correspondencia
}

Recibido Ene. 20, 2015; Aceptado Mar. 29, 2015; Versión final Jun. 6, 2015, Publicado Oct. 2015

\begin{abstract}
Resumen
El objetivo de la presente investigación fue determinar el contenido de etanol, metanol y metales pesados en el ñeque, una bebida alcohólica a base de caña de azúcar producida artesanalmente en la Costa Caribe colombiana. Para la elaboración de ñeque se disuelve panela (zumo de caña de azúcar) en agua hasta llegar a una densidad de un grado Baumé. Esta solución se fermenta aproximadamente siete días, obteniendo un mosto. Luego se destila obteniéndose como destilado la bebida alcohólica. El contenido de etanol y metanol se determinó por cromatografía de gases. La identificación de metales se utilizó mediante la metodología de plasma acoplado inductivamente a espectroscopia de emisión atómica. Las concentraciones de etanol, cobre y plomo están por sobre los límites permisibles para este tipo de bebidas alcohólicas.
\end{abstract}

Palabras claves: ñeque, bebidas alcohólicas, bebida artesanal, metanol, metales pesados

\section{Characterization of Ñeque, a Home-made Alcoholic Beverage Produced in the Colombian Caribbean Coast}

\begin{abstract}
The objective of this research was to determine the content of ethanol, methanol and heavy metals in ñeque, an alcoholic beverage obtained from sugarcane in a home-made form in the Colombian Caribbean Coast. To prepare ñeque, panela (sugar cane juices) is dissolved in water until a density of one Baume degree is reached. This solution is fermented approximately for seven days obtaining a must. After that, the must is distilled obtaining the alcoholic beverage as distillate. The ethanol and methanol content was determined by gas chromatography. The identification of metals was done by inductively coupled plasma atomic emission spectroscopy. Ethanol concentrations, copper and lead are higher than the permissible limits for this type of alcoholic beverages.
\end{abstract}

Keywords: ñeque, alcoholic beverage, alcoholic beverage, methanol, heavy metals 


\section{INTRODUCCIÓN}

En las últimas décadas el abuso del alcohol y el alcoholismo han cobrado gran importancia a nivel mundial, debido a sus consecuencias sociales y sanitarias (López-Naranjo et al., 2013). El consumo de alcohol causa 2 millones 500 mil muertes cada año, y se relaciona con las causas de muerte de 320 mil jóvenes de entre 15 y 29 años de edad, lo que representa un $9 \%$ de las defunciones en ese grupo de edad (Lachenmeier et al., 2008; 2009; López-Naranjo et al., 2013). Asimismo, el alcoholismo ocupa el tercer lugar entre los factores de riesgo de la carga mundial de morbilidad; es el primer factor de riesgo en el Pacífico Occidental y las Américas, y el segundo en Europa (López-Naranjo et al., 2013).

El chirrinchi, chirrinche o tapa'etuza (en alusión a la tusa de maíz usada con frecuencia para tapa de su envase), es un aguardiente artesanal elaborado mediante un proceso de fermentación y destilación. Es destilado clandestina y artesanalmente a partir de mostos de sumo de caña de azúcar o panela sometidos a fermentación alcohólica, y se consume en algunas regiones campesinas (NTC 410, 1999; Plutowska y Wardencki, 2008; Cabrera, 2009; Chaves-López et al., 2014). Estas bebidas alcohólicas (BA) se consumen ampliamente en los departamentos de Sucre, Guajira, Cesar, sur del Magdalena y zona central de Bolívar, donde particularmente recibe el nombre de ñeque, y se caracterizan por altos contenidos de metanol y metales pesados (Plutowska y Wardencki, 2008; López-Naranjo et al., 2013). Los instrumentos y aparatos utilizados en la elaboración de esta BA son: la múcura (tinaja), pesajarabe (densímetro), pesalicor (alcoholímetro) y alambique (aparato de destilación provisto con serpentín para la condensación de los vapores) (Cabrera, 2009). Las materias primas utilizadas son: agua, panela y yerbas aromáticas como el anís (utilizado como saborizante).

Estas BA de fabricación artesanal, se obtienen por el proceso de fermentación de los jugos ricos en carbohidratos (principalmente sacarosa) extraídos de la caña de azúcar; y en Colombia no existe regulación alguna para su fabricación, pues no posee registro sanitario del Instituto Nacional de Vigilancia de Medicamentos y Alimentos (INVIMA), y por lo tanto no cumple con las normas establecidas por el Instituto Colombiano de Normas Técnicas (ICONTEC) (Olarte et al., 2007). Los niveles de alcohol en ñeque son variables según el proceso de elaboración en las distintas regiones (Olarte et al., 2007). Una característica importante del consumo de este tipo de BA, es su inicio desde la infancia en poblaciones campesinas, donde se bebe habitualmente durante las jornadas diarias de trabajo en el campo, y por lo cual el riesgo de desarrollo de cirrosis por alcohol se presenta en edades más tempranas que en las poblaciones de las ciudades industrializadas, donde el consumo de alcohol está restringido a los menores de edad y por lo tanto el riesgo de hepatopatía por alcohol se presenta en edades más avanzadas (Idrovo, 2007; Olarte et al., 2007).

Las principales causas de muerte globalmente atribuibles al consumo de BA artesanales son: cirrosis hepática, cáncer de hígado, cáncer de esófago, enfermedades del corazón, enfermedades cerebrovasculares, cánceres oro-faríngeos, entre otras (López-Naranjo et al., 2013). El metanol se conoce como alcohol de madera, y está presente en diferentes procesos de fabricación de BA como parte normal del proceso de fermentación, encontrándose en mayor o menor proporción (trazas). La toxicidad por ingesta de metanol se debe a que es metabolizado por oxidación a formaldehido y en una segunda oxidación hasta ácido fórmico, que afecta a las células retinianas, cardíacas, hepáticas y encefálicas. La excreción de metanol es más lenta que la del etanol (Roldan et al., 2003), presenta un efecto mucho más nocivo para el ser humano y contribuye a desarrollar distintas afecciones tales como: trastornos neurológicos, cirrosis hepática, enfermedades cardiovasculares, ceguera, alteraciones genéticas, hemorragias gastrointestinales, cáncer en cualquier parte del tracto digestivo o incluso la muerte por la alta y excesiva ingestión de BA adulteradas con una dosis letal de 60 a 250mL (Alcalá, 2002; García y López, 2004; López-Naranjo et al., 2013). En Colombia las BA ilegales representan el $23,6 \%$ del total del mercado en términos de volumen y el $12,1 \%$ en términos de valor, el mercado legal representa el otro $76,4 \%$ y $87,9 \%$ respectivamente. Usualmente, durante el procesamiento de estas BA ilegales, la cabeza de la destilación no se separa del resto del destilado; y esta cabeza es donde se encuentra la mayor cantidad de metanol (Cabrera, 2009). Por lo anterior, se hace necesario poner en aviso público el contenido de metanol en el ñeque, con el ánimo de promover estrategias de control adecuadas que ayuden a minimizar su consumo.

Aunque algunos minerales son parte natural del cuerpo humano, cuando rebasan las concentraciones homeostáticas se transforman en unos de los contaminantes más peligrosos y tóxicos para el organismo (López-Naranjo et al., 2013). Casi todos los metales son tóxicos en concentraciones altas y algunos de ellos constituyen venenos graves incluso a concentraciones bajas. Ejemplo de los anterior son el cobre y el plomo, los cuales no deben estar presentes como contaminantes en alimentos, agua purificada, agua potable y BA (López-Naranjo et al., 2013). Al igual que en el caso del metanol, la bioacumulación de dichos metales en el organismo humano como consecuencia del consumo nocivo de ñeque puede ocasionar serios problemas de salud pública. Consecuentemente, en las BA como el ñeque es necesario analizar el contenido de los metales 
mencionados, y es deber de los entes reguladores crear normas oficiales para reconocer su daño potencial a la salud de los individuos (López-Naranjo et al., 2013; Szymczycha-Madeja et al., 2015).

En Colombia Olarte et al., (2007) determinó la concentración de etanol, acetaldehído y metanol en el guarapo consumido por pacientes con cirrosis hepática alcohólica en el departamento de Cundinamarca, encontrando que esta bebida tradicional presentó niveles significativos de etanol, y que además su consumo en grandes cantidades y por largo tiempo supera ampliamente el umbral tóxico asociado al desarrollo de cirrosis hepática. Sin embargo la presente contribución es el primer estudio con este enfoque para bebidas locales como el ñeque, y el conocer esta información permitirá establecer políticas sanitarias para la población regional y la nación, el objetivo del presente trabajo fue determinar el contenido de etanol, metanol y metales pesados en el ñeque producido en la Costa Caribe colombiana.

\section{MATERIALES Y MÉTODOS}

\section{Elaboración del ñeque}

La panela se disolvió en agua pura hasta llegar a una densidad de un grado (1ํ) Baumé, la cual se corroboró con el densímetro. Después se sometió a fermentación por siete días (Granados et al., 2013), y a medida que pasaban los días se observó efervescencia (espumas) producida por el dióxido de carbono que se formaba. El punto ideal en el cual se realizó la destilación fue cuando el densímetro marcó cero en grados $\left(0^{\circ}\right)$ Baumé, indicando que la fermentación había terminado y que el mosto estaba listo. Las condiciones de fermentación reportadas para este tipo de bebidas artesanales dependen de la zona de producción, y pueden estar en rangos de $\mathrm{pH}$ de 4 a 6 y temperaturas entre 30 y $40^{\circ} \mathrm{C}$. Las concentraciones de inóculo pueden ser variadas y no tenidas en cuentas, debido a lo artesanal y empírico del proceso (Ferreyra et al., 2009; Andrade et al., 2009). Seguidamente, el mosto se depositó en una paila (balón de fondo redondo con desprendimiento lateral construido en latón), sometiéndose a calentamiento con el fin de realizar una separación del alcohol de la mezcla en forma de vapor en el serpentín, lugar donde se condensaron los vapores, obteniendo un producto mezcla de la cabeza y el restos del destilado, que fueron recogidos en un recipiente de vidrio. Debido a que el material donde se realiza la destilación es una aleación de cobre, durante el calentamiento del líquido algo de este elemento logra ser arrastrado en la evaporación (Olarte et al., 2007). Localmente a este ron se le llama ron ñeque, Churrinche o tapa'etuza y es distribuido en diferentes recipientes de distinta cantidad y valor.

\section{Determinación de contenido de etanol y metanol}

El contenido de etanol en ñeque se determinó por medio de cromatografía de gases (AgilentTechnologies 7890A, acoplado a un espectrómetro de masas Agilent Technologies 5975C equipado con un puerto de inyección Split/splitlles (relación 1:50), un inyector automático Agilent 7863 y un sistema de datos HP Chem Station.), el cual se utilizó para la identificación y cuantificación de metanol en ñeque. El análisis se realizó por duplicado en cada muestra. El Gas portador fue nitrógeno a $20 \mathrm{~mL} / \mathrm{min}$., se trabajó a una temperatura de horno $85^{\circ} \mathrm{C}$, temperatura inicial $50^{\circ} \mathrm{C}$, rampa de temperatura $10^{\circ} \mathrm{C} / \mathrm{min}$ hasta $80^{\circ} \mathrm{C}$, temperatura del inyector y del detector $250^{\circ} \mathrm{C}$. Luego se estableció una curva de calibración partiendo de un estándar. Al final se tomó muestras de aguardiente fermentado (ñeque) y se inyectó directamente al equipo determinando cuales eran las concentraciones de metanol y etanol.

\section{Preparación de la muestra y estándar}

Se realizó la metodología descrita por la guía componente práctico de la Universidad Nacional Abierta y a Distancia (2014). Se tomaron $10 \mathrm{~mL}$ de la muestra a $40^{\circ} \mathrm{C}$ y se llevó a volumen con agua destilada en un balón de $100 \mathrm{~mL}$, al instante se filtró la solución con papel Whatmann No. 4. Para la preparación del estándar se tomó $1 \mathrm{~mL}$ de metanol y $1 \mathrm{~mL}$ de etanol y se llevó a volumen con agua destilada en dos balones de $100 \mathrm{~mL}$, respectivamente.

\section{Cálculos}

Finalmente se calcularon las áreas relativas de cada una de las muestras analizadas mediante la Ecuación (1). Las áreas relativas encontradas se interpolaron en la curva de calibración, corregida previamente con regresión lineal, y de esta manera se obtuvo las concentraciones de metanol en las muestras analizadas en $\mathrm{mg} / 100 \mathrm{~mL}$.

$$
\text { Área relativa }=\frac{\text { Área de la muestra }}{\text { Área del estándar interno }}
$$




\section{Determinación de cobre $(\mathrm{Cu})$ y plomo $(\mathrm{Pb})$}

Se utilizó plasma acoplado inductivamente a espectroscopia de emisión atómica (ICP-AES) según la metodología descrita por Terol et al., (2011).

\section{ANÁLISIS DE RESULTADOS}

En la Tabla 1 se observan los contenidos de etanol, metanol, cobre y plomo en las BA fermentadas preparadas en corregimientos y municipios del Departamento de Bolívar, y se comparan con los niveles permitidos por la Norma Técnica Colombiana (NTC) 410 (1999), la cual dicta los requisitos específicos de BA a base de caña de azúcar como el ñeque de este estudio. El etanol, uno de los componentes principales de las BA, se puede obtener por fermentación o destilación, y se consideran dosis toxicas de etanol en adultos en cantidades de $5 \mathrm{~g} / \mathrm{Kg}$ y en niños de $3 \mathrm{~g} / \mathrm{Kg}$ (Roldan et al., 2003). La OMS recomienda beber menos de $60 \mathrm{~g}$ de etanol en hombres y $40 \mathrm{~g}$ de etanol en mujeres (Chang y Barroso, 2005; Téllez y Cote, 2006). En un estudio realizado en la calidad de varias BA comercializadas en México, se pudo establecer que el contenido de etanol (\%) para el ron estuvo en promedio desde 28 a 35\% y para tequila alrededor del 38\% (López-Naranjo et al., 2013), teniendo así una similitud con el ñeque comercializado en Turbaco, Ballesta y Rocha.

En la Tabla 1 se puede evidenciar que ningún ñeque alcanza el contenido mínimo de etanol según la NTC 410 (1999). Este bajo contenido de etanol puede tener distintas implicaciones para la salud de los individuos; ya que según López-Naranjo et al., (2013) el individuo ingerirá mucho más alcohol en las BA hasta obtener los resultados de alcoholismo que espera o desea obtener. Por los niveles encontrados de etanol en ñeque de los corregimientos y municipios del Departamento de Bolívar, estas podrían no ser consideradas como aguardiente por las instituciones reguladoras para tal fin como el INVIMA, y no estarían definidas en las normas ICONTEC de buenas prácticas de manufactura. Lo anterior se relaciona con la falta de estudios sobre BA tradicionales ya que su elaboración y venta no requieren procesos de industrialización y comercialización que obliguen a cumplir con los requerimientos exigidos por la ley para este tipo de BA, es por esto que, de acuerdo a las normas de ICONTEC el ñeque podría ser considerado como una BA fraudulenta (Olarte et al., 2007).

Tabla 1: Contenidos de etanol, metanol, cobre y plomo de ñeque

\begin{tabular}{|c|c|c|c|c|}
\hline Neque Producido & $\%$ Etanol & Metanol $(\mathrm{mg} / \mathrm{L})$ & Cobre $(\mathrm{mg} / \mathrm{L})$ & Plomo $(\mathrm{mg} / \mathrm{L})$ \\
\hline Rocha & $31,0 \pm 1,23$ & $280 \pm 2,45$ & $4,0 \pm 0,34$ & $0,60 \pm 0,01$ \\
\hline Ballesta & $29,0 \pm 1,76$ & $90 \pm 1,54$ & $3,0 \pm 0,12$ & $0,70 \pm 0,00$ \\
\hline Puerto Badel & $27,0 \pm 1,72$ & $250 \pm 3,45$ & $2,0 \pm 0,06$ & $0,60 \pm 0,02$ \\
\hline Santa Rosa & $24,0 \pm 1,03$ & $150 \pm 2,43$ & $2,0 \pm 0,15$ & $0,50 \pm 0,00$ \\
\hline Villanueva & $31,0 \pm 1,00$ & $130 \pm 2,34$ & $1,0 \pm 0,09$ & $0,70 \pm 0,02$ \\
\hline Turbaco & $28,0 \pm 1,02$ & $80 \pm 1,89$ & $2,3 \pm 0,04$ & $0,40 \pm 0,00$ \\
\hline San Basilio Palenque & $25,0 \pm 1,83$ & $240 \pm 2,85$ & $3,2 \pm 0,08$ & $0,70 \pm 0,01$ \\
\hline María la Baja & $26,0 \pm 1,73$ & $260 \pm 2,56$ & $2,6 \pm 0,06$ & $0,60 \pm 0,03$ \\
\hline Arroyo Hondo & $25,7 \pm 1,72$ & $160 \pm 1,87$ & $2,2 \pm 0,05$ & $0,74 \pm 0,00$ \\
\hline Pasacaballos & $24,6 \pm 0,98$ & $270 \pm 2,93$ & $2,2 \pm 0,8$ & $0,76 \pm 0,01$ \\
\hline Permitido (NTC 410, 1999) & Mínimo 38 & Máximo 300 & Máximo 1 & - \\
\hline
\end{tabular}

La contaminación con metanol se origina cuando se produce la fermentación de jugos azucarados utilizados para la obtención de BA, en la cual, además de etanol, se producen también cantidades variables de metanol y demás compuestos volátiles (Olarte et al., 2007; López-Naranjo et al., 2013; Silva et al., 2013). Como se evidencia en la Tabla 1 los valores de metanol en ñeque de los corregimientos y municipios del Departamento de Bolívar no sobrepasan los límites permisibles nacionales (máximo $300 \mathrm{mg} / \mathrm{L}$ según NTC 410) ni internacionales (máximo 500mg/L) (Ministerio de Salud, 2001). Resultados similares encontraron otros autores al no hallar valores de metanol en BA por encima de las Normas locales (Olarte et al., 2007; LópezNaranjo et al., 2013). Sin embargo, estos valores están estrechamente cercanos al límite máximo, por lo cual se debe estar alerta, teniendo en cuenta la posible bioacumulación de este material en el organismo humano como consecuencia del consumo constante de ñeque con estos altos contenidos de metanol, que podrían ocasionar serios problemas en la salud del consumidor (Olarte et al., 2007; López-Naranjo et al., 2013), entre los que se encuentran el daño renal, alteración cerebral y ceguera (Olarte et al., 2007; Péres et al., 2012; López-Naranjo et al., 2013). Estos altos contenidos de metanol son debidos a que durante el procesamiento del ñeque en los corregimientos y municipios de Bolívar, la cabeza de la destilación no se separa del resto del destilado; y esta cabeza es donde se encuentra la mayor cantidad de metanol (Cabrera, 2009).

Referente al contenido en cubre, el ñeque de corregimientos y municipios del Departamento de Bolívar sobrepasan el límite máximo permisible para este tipo de BA (mg/L según NTC 410), y en algunos como San 
Basilio Palenque, Rocha y Ballesta alcanza valores alarmantes. Estos altos contenidos se deben dado que el material del cual se construyen los calderines de destilación son aleaciones de cobre, y en el calentamiento del líquido algo de este elemento logra ser arrastrado por los vapores (Olarte et al., 2007; Cabrera, 2009). En rones elaborados artesanalmente en Brasil, la presencia de cobre en Cachaça (aguardiente de caña de azúcar), según la legislación de Brasil puede tener un límite máximo de $5 \mathrm{mg} / \mathrm{L}$ de cobre, sin embargo los límites de rones identificados tuvieron un rango de 8,08 a $11,82 \mathrm{mg} / \mathrm{L}$ (Do Nascimiento et al., 2008), lo cual muestra el gran riesgo en salud de quienes consumen estas BA con tan altos contenidos del metal. Es de resaltar que al igual que en el caso del metanol, la bioacumulación de este metal en el organismo humano como consecuencia del consumo nocivo de ñeque puede ocasionar serios problemas de salud pública (LópezNaranjo et al., 2013).

La legislación colombiana no regula el contenido de plomo en aguardientes, sin embargo, en Europa se establece que el nivel de plomo permitido es de $0,3 \mathrm{mg} / \mathrm{L}$, en cambio en EE.UU y Canadá es de 0,1 a 0,2mg/L respectivamente (Petit de Peña et al., 2004), por lo cual los niveles de plomo para el ñeque de los corregimientos y municipios del Departamento de Bolívar están fuera de los parámetros de calidad internacionales. Nuevamente este es un dato preocupante, ya que la presencia de plomo en estas concentraciones es inaceptable debido a que estos metales pesados impiden la absorción de vitaminas y de otros minerales en el organismo (López-Naranjo et al., 2013). En otros estudios Petit de Peña et al., (2004) determinaron el contenido de plomo en 28 BA artesanales en Brasil, apreciando que las cantidades de plomo en las diferentes muestras variaron desde 12,6 a $370 \mu \mathrm{g} / \mathrm{L}$ con un valor promedio de $78,9 \mu \mathrm{g} / \mathrm{L}$. Aunque las muestras se produjeron a partir de la misma base de fermentación siempre hay diferencias muy elevadas en el contenido de este elemento debido a que el proceso de destilación es llevado a cabo por productores artesanales independientes.

\section{CONCLUSIONES}

Los resultados obtenidos muestran que todas las muestras analizadas poseen un contenido de etanol por debajo de lo que la norma colombiana respectiva permite; un contenido de metanol por debajo de lo permisible, pero contenidos de cobre y plomo por arriba de lo permisible incluso por normas vigentes en Europa y en Estados Unidos de América. Se destaca que la información obtenida de estudio puede ser utilizada para modificar las regulaciones existentes sobre la producción y consumo de esta bebida y así como de las implicaciones sobre la salud, en lo que toca al efecto de metanol y metales presentes.

\section{REFERENCIAS}

Alcalá, J.N., Intoxicación por metanol, Anales de Medicina Interna, 19(9), 494-495 (2002)

Andrade, R. y otros cuatro autores, Obtención de aguardiente a partir de batata (Ipomoea batatas), Temas Agrarios, 14(1), 39-45 (2009)

Cabrera, F., Bebidas fermentadas, Unidad de Ciencias básicas, tecnología e ingeniería, Universidad Nacional Abierta y a Distancia: UNAD, Bogotá D.C. (2009). http://datateca.unad.edu.co/contenidos/306598/ contLinea/index.html. Acceso: 25 de Diciembre (2014)

Chang de la Rosa, M., M. Canizares y I. Barroso, Consumo de bebidas alcohólicas: Sugerencias para su medición en Cuba, Revista Cubana de Medicina General Integral, 21(3-4), 1-11 (2005)

Chaves-López, C. y otros cinco autores, Traditional fermented foods and beverages from a microbiological and nutritional perspective: The colombian heritage, Comprehensive Reviews in Food Science and Food Safety, 13(5), 1031-1048 (2014)

Do Nascimento, R. y otros cinco autores, Determinación espectrofotométricos de cobre en el azúcar de caña usando espíritu biquinolina en presencia de etanol y Triton X-100, Spectrochimica Acta Part A: Molecular and Biomolecular Spectroscopy, 71(4), 1414-1418 (2008)

Ferreyra, M.M. y otros cinco autores, Fermentación alcohólica de jugo de naranja con S. cerevisiae, Ciencia, docencia y tecnología, 39, 143-158 (2009)

García, G.M. y M.A. López, Bebidas alcohólicas no destiladas. Biotecnología alimentaria, 5ta reimpresión, 1a Ed., p. 263. Editorial Limusa, México D.F., México (2004)

Granados, C. y otros tres autores, Evaluación fisicoquímica y microbiológica del aperitivo vínico de lulo (Solanum quitoense L.), Información Tecnológica, 24(6), 35-40 (2013) 
Idrovo, V., Cirrosis y Bebidas alcohólicas artesanales, Revista Colombiana de Gastroenterología, 22(2), 82 $82(2007)$

Lachenmeier, D.W., B. Sarsh y J. Rhm, The composition of alcohol products from markets in Lithuania and Hungary, and potential health consequences: A pilot study, Alcohol Alcohol, 44 (1), 93-102 (2009)

Lachenmeiera, D.W., S. Haupta y K. Schulzb, Defining maximum levels of higher alcohols in alcoholic beverages and surrogate alcohol products, Regulatory Toxicology and Pharmacology, 50(3), 313-321 (2008)

López-Naranjo, F. y otros cuatro autores, La calidad de varias bebidas alcohólicas comercializadas en México y las consecuencias potenciales en la salud pública, Revista Mexicana de Ciencias Farmacéuticas, 44(4), 62$72(2013)$

Ministerio de Salud, Evaluación de riesgos: bebidas alcohólicas artesanales, Serie de Informes Técnicos de Vigilancia Epidemiológica de Riesgos y Daños №27, 82p. Lima, Perú (2001)

NTC 410. Instituto Colombiano de Normas Técnicas y Certificación (Colombia). Bebidas alcohólicas. Aguardiente de caña. Caña, cachaza o branquiña. Sexta Actualización, Bogotá, Colombia (1999)

Olarte, M.F. y otros tres autores, Determinación de los niveles de etanol, metanol y acetaldehído en el guarapo elaborado en los municipios de Cundinamarca, Revista Colombiana de Gastroenterología, 22(2), 97-103 (2007)

Péres, L.O. y otros cuatro autores, Conductive polymer gas sensor for quantitative detection of methanol in Brazilian sugar-cane spirit, Food Chemistry, 130(4), 1105-1107 (2012)

Petit de Peña, A., y otros seis autores, Continuous flow system for lead determination by faas in spirituous beverages with solid phase extraction and on-line copper removal, Talanta, 64(5), 1351-1358 (2004)

Plutowska, B. y W. Wardencki, Application of gas chromatography-olfactometry (GC-O) in analysis and quality assessment of alcoholic beverages - A review. Food Chemistry, 107(1), 449-463 (2008)

Roldan, J., C. Frauca y A. Duenas, Intoxicación por alcoholes, Anales del Sistema Sanitario de Navarra, 26(1), 129-139 (2003)

Silva, R.O. M. Batistote y M.P. Cereda, Alcoholic fermentation by the wild yeasts under thermal, osmotic and ethanol stress, Brazilian Archives of Biology and Technology, 56(2), 161-169 (2013)

Szymczycha-Madeja, A. y otros cuatro autores, Advances in assessing the elemental composition of distilled spirits using atomic spectrometry, TrAC Trends in Analytical Chemistry, 64, 127-135 (2015)

Téllez, J. y M. Cote, Alcohol etílico: Un tóxico de alto riesgo para la salud humana socialmente aceptado, Rev Revista de la Facultad de Medicina, 54(1), $32-47$ (2006)

Terol, A. y otros cuatro autores, Alcohol and metal determination in alcoholic beverages through hightemperature liquid-chromatography coupled to an inductively coupled plasma atomic emission spectrometer, Journal of Chromatography A, 1218(22), 3439-3446 (2011) 